\title{
The Use of Brillouin Enhanced Four Wave Mixing for Phase Conjugation of Laser Radiation
}

\author{
Oleg G. KOTIAEV*, Shigeaki UCHIDA*, and Hiroshi SAWADA** \\ * Institute for Laser Technology, 2-6 Yamada-oka, Suita, Osaka 565-0871, Japan \\ **University of Rochester, Dept. of Mechanical Engineering, Rochester, NY, 14627, USA
}

(Received June 14, 2001)

\begin{abstract}
Possibility of phase conjugation (PC) of solid-state laser radiation via Brillouin Enhanced Four-Wave Mixing (BEFWM) has been shown experimentally. Correction of various types of optical aberration introduced into incoming signal has been observed. PC-fidelity has been estimated to be equal to 0.62 . Principle of operation of a multifunctional telescope system using BEFWM effect for correction for primary mirror aberration is briefly described.
\end{abstract}

Key Words: Phase conjugation, Fidelity, Energy delivery, Multifunctional system, BEFWM

\section{Introduction}

Phase conjugation (PC) of laser radiation is considered as the most promising approach of nonlinear adaptive optics to the creation of high quality laser beams. Phase conjugation allows to correct laser beam distortions caused by aberrations of optical elements, thermal deformation, vibration etc. As a result, high quality laser beam can be formed and used, for example, for precise delivery of laser energy to remote objects or/and formation of undistorted image.

The final goal of the present study is to develop the principles of a laser system which makes it possible both observing of remote objects and delivering of energy with the use of one and the same telescope system. The presence of optical aberrations in the system results in degradation of image and decrease of radiation delivery efficiency. The effect of phase conjugation of laser radiation is supposed to be used for correction of phase distortions caused by optical aberrations. Taking into account that a pulsed solid-state Nd:YAG laser operating at wavelength of $1 \mu \mathrm{m}$ is supposed to be used as a source of laser radiation, it is necessary to choose an appropriate mechanism of phase conjugation. At present time, one of the most attractive mechanisms of phase conjugation of near infrared radiation is based on the process of Brillouin Enhanced Four-Wave Mixing (BEFWM).

The effect BEFWM is known by capability of phase conjugation of extremely weak signals (as low as $10^{-14} \mathrm{~J}$ ) with a large reflection coefficient (up to $10^{6}$ ). ${ }^{1-5}$ ) There are a number of publications demonstrating high effective BEFWM mirrors. However, there is explicit lack of the reports about a PC capability. The present paper describes the experimental examination of the PC of laser radiation by means of BEFWM and demonstration of correction for wave front distortions.

\section{Brillouin enhanced four-wave mixing effect}

The technique of BEFWM is just a form of nearly degenerate four wave mixing (Fig.1). The mixing and interaction occur inside a cell filled with nonlinear medium which is characterized by Brillouin nonlinearity, i.e. can be used for realization of Stimulated Brillouin Scattering (SBS) of laser radiaton. The incoming signal to be conjugated interacts with coherent backward pump beam which has a Brillouin frequency shift with respect to the incoming signal, so that the produced interference pattern moves at close to acoustic velocity and drives an acoustic (hypersonic) wave. The acoustic wave induced by moving interference pattern acts as a density grating or dynamic hologram. The wave scatters the forward pump beam to form the conjugate beam. Interference between scattered (conjugated) beam and forward pump results in amplification of the acoustic wave and increase of its efficiency in scattering the forward pump beam. The acoustic wave is amplified and its efficiency in scattering increases until a significant fraction of available power is extracted from the forward pump beam into the conjugated beam. As a result, power of the conjugated beam can reach the order of the forward pump power magnitude.

The effect of phase conjugation should take place during the scattering process. That means that the scattered beam has the same wave front as the incoming signal and propagates in the opposite direction. When the incoming signal to be conjugated

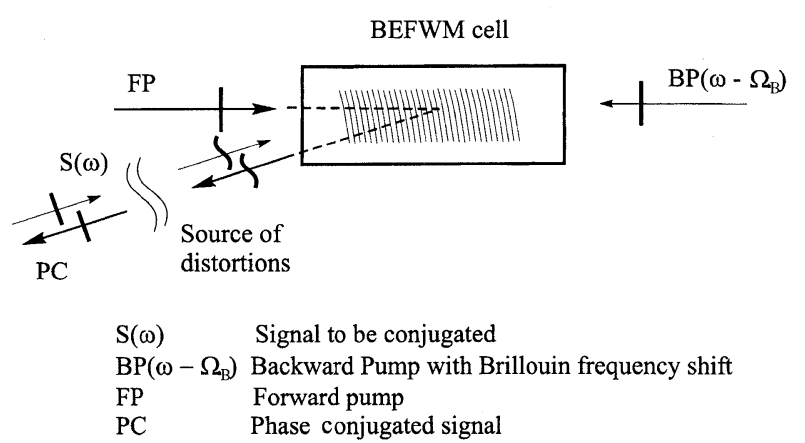

Fig.1 Geometry of Brillouin Enhanced Four-Wave Mixing effect. 
passes through a source of distortions (e.g. aberrated primary mirror of a telescope) quality of its wave front becomes very low. Due to the effect of phase conjugation, a beam with the reproduced phase distribution propagating in opposite direction appears. This beam is called phase conjugated signal. When propagating through the same source of distortions in opposite direction, the wave front distortions are corrected automatically, resulting in formation of a high quality output beam. A beam forming optical system equipped with PC mirror can send laser radiation to remote objects much more effectively than a system without PC correction of phase distortions.

\section{Experimental setup}

The experimental setup shown in Fig. 2 is used for the characterization of the BEFWM process. The setup allows to determine energetic, spatial and temporal characteristics of laser signals. The main parameters subject to study are PC fidelity, energy efficiency (or reflection coefficient of PC mirror) and phase conjugation dynamics.

A Nd:YAG laser assembled on the Master-Oscillator-Power Amplifier (MOPA) scheme is used as a source of laser radiation. Output radiation energy achieves $1 \mathrm{~J}$ in 22-ns pulse (FWHM). Beam size is equal to $15 \mathrm{~mm}$. Output telescope T with magnification $M=0.4$ is used for reducing the beam size and collimating the output radiation. Output radiation is polarized in a horizontal plane.

Five percent fraction of the output radiation reflected by beamsplitter BS1 is used as a signal to be conjugated $\mathrm{S}$. The rest of the radiation is brought into an SBS cell for forming the pump waves: forward pump FP and backward pump BP. Liquid fluorocarbon FC-72 is used as an SBS medium. The main properties of the medium are the following:

- refractive index $n=1.248$,

- sound velocity $v_{\mathrm{s}}=512 \mathrm{~m} / \mathrm{s}$,

- Brillouin frequency $\Delta v_{\mathrm{B}}=1.2 \mathrm{GHz}$. $^{6,7)}$

Length of the SBS cell is equal to $30 \mathrm{~cm}$. Radiation is focused by lens $\mathrm{L} 1$ with a focal length of $F=30 \mathrm{~cm}$ into the depth of $25 \mathrm{~cm}$. SBS reflectivity achieves the value of $85 \%$. Quarter wave plate QWP1 installed in front of the SBS cell provides

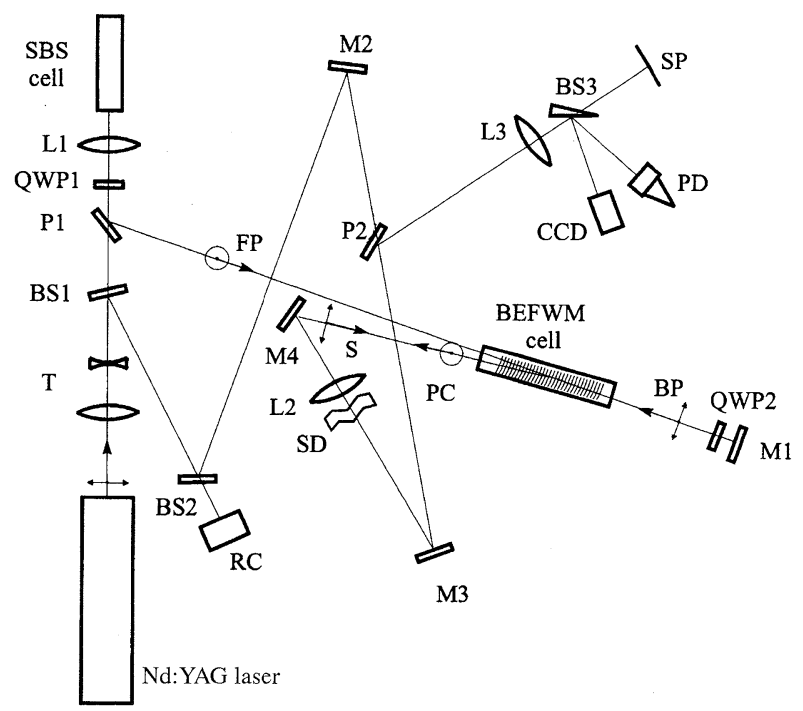

Fig.2 Experimental setup for measuring the fidelity of phase conjugation. rotation of polarization plane of the reflected beam by $90^{\circ}$.

The SBS cell is used for providing Brillouin shift to frequency of the pump waves. It is necessary for performing BEFWM process. After reflecting off the SBS cell, radiation is reflected by polarizer P1 and brought into the BEFWM cell of $20 \mathrm{~cm}$ length as a forward pump FP. Mirror M1 and quarter wave plate QWP2 form a backward pump BP (polarized in a horizontal plane as the signal to be conjugated). A ratio of the forward pump intensity to the backward pump intensity is $15: 1$. Beam size of both pumps is equal to $6 \mathrm{~mm}$. Temporal shift between the pump waves is not considerable: less than $5 \%$ of the laser pulse duration.

The signal to be conjugated is brought into the BEFWM cell after reflecting off the calorimeter beamsplitter BS2 $(R=20 \%)$ and passing through the optical line M2 - M4. The optical line provides zero optical path difference between the signal and backward pump. In accordance with the condition of beam size ratio required for high-fidelity phase conjugation, ${ }^{1)}$ the signal beam size is reduced by lens L2. Resulting signal beam size is 3 times less than the pump beam size.

Intersection angle inside the BEFWM cell $\theta$ is equal to $\pi$ 0.014 radians. That means that the signal to be conjugated and the backward pump form an interference pattern with spatial period of $d=1 / 2 n \sin (\theta / 2)=0.43 \mu \mathrm{m}$ inside the BEFWM cell. Because of frequency shift this pattern moves at close to sound velocity and induces an appropriate acoustic wave. The acoustic wave functions as a density grating or dynamic hologram. Forward pump is scattered by this grating producing the phase conjugation signal (PC signal). Since the forward pump is polarized in a vertical plane, the PC signal is also polarized in a vertical plane. It is reflected by polarizer $\mathrm{P} 2$ and brought into detecting scheme which consists of CCD-camera, Pin-diode PD and thermal sensitive paper SP. Lens L3 forms a far field zone at the sensing head of the CCD-camera. Angular size of the sensing head is equal to $12 \times 9 \mathrm{mrad}$ that approximately corresponds to $24 \times 18$ diffraction limits of the incoming beam.

Energy of the signal to be conjugated and pump beams is measured by the reference calorimeter RC.

\section{Experimental results}

Before testing PC fidelity, possibility of spurious SBS of the signal to be conjugated in the BEFWM-cell has been checked. No spurious SBS has been detected. That means that intensity of the signal to be conjugated is under SBS threshold at the present conditions and the PC signal totally is due to BEFWM process.

To test PC fidelity, the signal to be conjugated passes through a source of phase distortions SD installed in front of lens L2. Optical elements characterized by various types of aberrations are used as the distortion sources. The left column of Fig.3 represents far field intensity distribution of the signal to be conjugated with various types of phase aberrations, and the right column represents the corrected signal-phase conjugated signal passed through the same source of distortion in opposite direction:

a) pure signal with no aberrations, the incoming beam with original quality is located at the center of the sensing head;

b) wedge-type aberration, the beam is shifted out of the center by the angle of $5 \mathrm{mrad}$, which is 10 times more than diffraction size of the incoming beam;

c) lens-type aberration simulated by additional lens with $F=5$ 
Aberrated signals

to be conjugated

a)

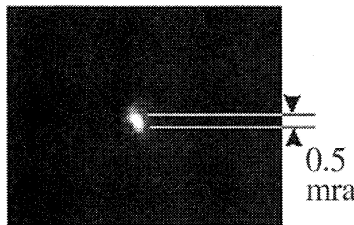

Corrected signals through phase conjugation

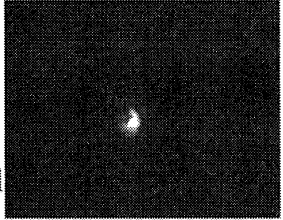

b)
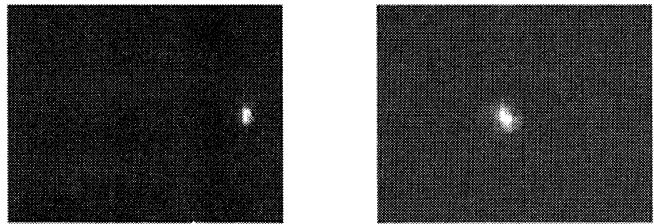

c)


d)
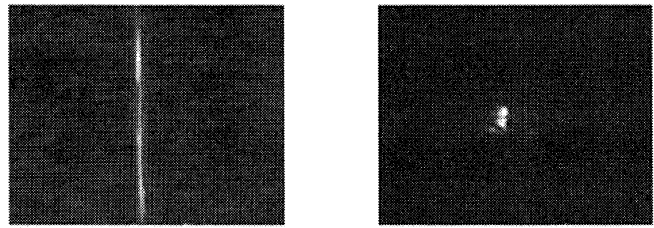

e)
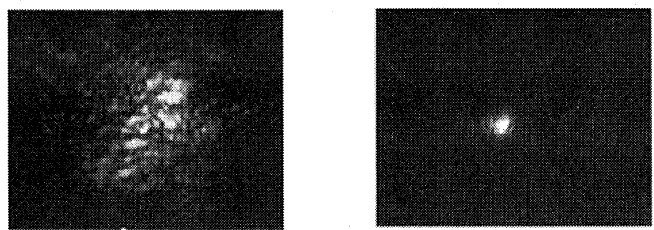

Fig.3 Far field intensity distribution of aberrated (left) and corrected (right) signals. Aberrations introduced: a) no aberration, b) wedge, c) lens, d) astigmatism, e) random phase aberration.

$\mathrm{m}$; the beam divergence is 4 times increased;

d) astigmatism simulated by cylindrical lens with $F=50 \mathrm{~cm}$; far field distribution has a form of vertical line;

e) random phase aberration simulated by random phase plate, the beam divergence is increased about 5 times, and far field distribution has speckle structure.

Phase conjugation of laser beam via BEFWM process has been realized. Pictures on the right column clearly demonstrate correction of aberration introduced into the signal to be conjugated. Estimation of PC fidelity is made in terms of laser beam divergence, since it defines divergence defines one of the most important properties of laser beam-directionality. There are two ways of measuring the divergence:

1) measuring the degree of beam spreading at the far field. If the far field is formed in the focal plane of a lens with focal length $\mathrm{F}$, and a laser beam diameter in the focal plane is $A$, then the angle of divergence $\theta=A / F$. It is convenient to compare the angle of divergence of the real beam with the angle of divergence of so called "ideal beam", which is characterized by perfect spatial coherence, plane wave front and constant amplitude over cross section. In the case ideal beam of circular cross section with diameter $D$, far field pattern due to diffraction consists of a circular central zone surrounded by a series of rings of rapidly decreasing intensity. Usually, the angle of divergence is defined in this case as an angular diameter of the first dark ring: $\theta=2.44 \lambda D$, where $\lambda$ - radiation wavelength. ${ }^{8)}$ This angle can be considered as a minimum possible, diffraction-limited divergence angle of a real beam of the same cross section.

2) measurement of a fraction of laser energy, which propagates within a certain solid angle. It is convenient to use the solid angle corresponding to the diffraction-limited angle of divergence of the ideal beam described above. For example, in the case of the ideal beam, fraction of laser energy $W_{\mathrm{dif}}$, which propagates within the diffraction-limited solid angle, is equal approximately to 0.84 . Of course, a real beam is characterized by lower value of $W_{\text {dif }}$.

Because the supposed laser system with correction for phase distortions is intended for effective energy delivery to remote (point) objects, the second way of definition is used. The fraction of laser energy $W_{\text {dif }}$, which propagates within diffractionlimited solid angle, is used as a parameter characterizing beam divergence. PC fidelity is estimated via comparison between divergence of phase conjugated beam passed through the source of distortion in opposite direction and divergence of incoming signal in front of the source of distortions. Actually, corresponding fractions of laser energy within diffraction-limited solid angle $W_{\text {dif }}(\mathrm{PC})$ and $W_{\text {dif }}(0)$, respectively, are compared. PC fidelity is defined as a ratio $Q_{\mathrm{PC}}=W_{\text {dif }}(\mathrm{PC}) / W_{\text {dif }}(0)$.

The energy fraction $W_{\text {dif }}(0)$ is evaluated as 0.69 . After passing through the source of distortions, the energy fraction within diffraction angle decreases significantly: from 0.08 at lens type

a)


c)



Fig.4 Pulse shapes of detected signals: a) incoming signal to be conjugated, b) pump pulses, c) phase-conjugated signal. 
aberration to 0 at wedge type aberration. However, after reflection off PC mirror and passing through the same source of distortion in the opposite direction, the considerable correction of phase distortions is observed. In the experiments, the energy fraction $W_{\mathrm{dif}}(\mathrm{PC})$ achieves the value 0.43 and remains approximately the same at any types of aberrations introduced into the incoming signal. That means that PC fidelity achieved the value $Q_{\mathrm{PC}}=0.43 / 0.69 \approx 0.62$.

In the present experiments, laser pulse shapes are detected. Figure 4 shows pulse shapes of the detected signals. Pulse duration of incoming signal (a), pump waves (b) and PC signal (c) are equal to $22.5,19.5$ and $10 \mathrm{~ns}$, respectively. Pulse shapes of all the signals have a bell form.

PC signal of $100 \mathrm{~mJ}$ is obtained at incoming beam energy of $20 \mathrm{~mJ}$. The reflectivity of the PC mirror achieves 500\%. Taking into account that pulse duration of the PC signal is two times shorter than one of the incoming signal, this value corresponds to $1000 \%$ power enhancement. It is estimated that up to $40 \%$ of forward pump energy is scattered producing PC signal.

\section{Discussion}

The fact that PC fidelity obtained in the experiments is less than unity $\left(Q_{\mathrm{PC}} \approx 0.62\right)$ is mostly explained by using the pump beams of quality not enough for high fidelity performance. As it was mentioned, energy fraction $W_{\text {dif }}(0)$ was evaluated as 0.69 . It should be noted that both the incoming signal and pump waves are characterized by this value. But in the case of ideal beam,
$W_{\text {dif }}($ ideal $) \approx 0.84$. As it is known, to realize high PC fidelity via four-wave mixing, the pump waves should be exactly conjugated to each other. For example, in the present experiments the pump waves are supposed to be formed as two high-quality counter-propagating plane waves. But the value $W_{\text {dif }}(0)=0.69$ means that quality of the pump waves is not high. As a result, PC fidelity is less than unity. The use of pump waves of higher quality will result in the increase of PC fidelity. Nevertheless, even with imperfect pump waves, the capability of correction for strong phase distortions is demonstrated. This important practical result verifies that BEFWM technique can be applied to the delivery mode of operation of multifunctional telescope system intended for effective energy delivery to various remote objects.

Strong pulse compression via SBS process is not observed in the experiments because of short interaction length. ${ }^{7)}$ As a result, the pulses of incoming signal and pump waves have approximately the same duration. But pulse duration of PC signal is approximately two times shorter than one of the incoming and pump beams. It can be explained by peculiarities of the dynamics of the BEFWM PC mirror. It takes a certain time to create the grating which scatters the laser radiation effectively. The reason is the necessity of exceeding the threshold intensity as well as some sluggishness of electrostriction force. Under the present experimental conditions, the creation time is approximately $10 \mathrm{~ns}$.

Comparatively low reflectivity of the PC mirror can be explained by using the incoming signal energy of tens $\mathrm{mJ}$ order. High reflectivity can he ohtained at much smaller incoming sig-

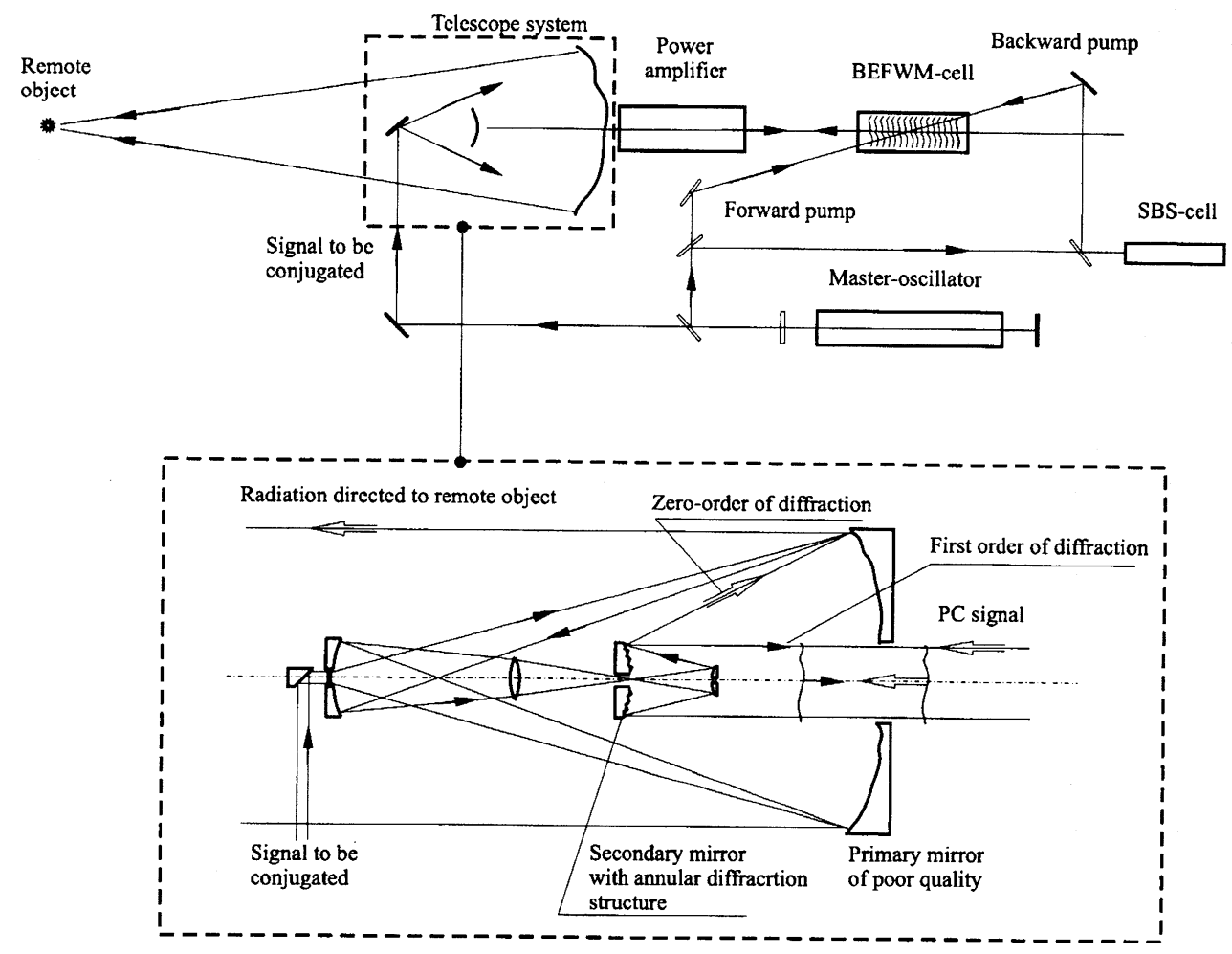

Fig.5 Schematic diagram of a multifunctional telescope system in the mode of energy delivery. The system is supposed to be equipped with the telescope system with nonlinear compensation of distortions. ${ }^{9)}$ Secondary mirror of the telescope system has annular diffraction structure on its surface. The signal to be conjugated in the direction of the first order of diffraction simulates signal coming from remote object: both signals have the same direction, the same wave front shape, as well as the same information of primary mirror aberrations. After procedure of phase conjugation, the PC signal in the direction of zero-order of diffraction is used. When reflecting from the primary mirror, wave front distortions caused by its aberrations are compensated, and corrected signal is directed to the remote object. 
nal energy (less than nanojoule).4)

Actually, not high reflectivity but absolute value of PC energy is the most important parameter of the supposed laser system using the BEFWM technique for precise delivery of laser radiation to remote objects. The principle of energy delivery mode of the system operation is shown in Fig.5. A telescope system in Fig.5 is equipped with a lightweight deployable membrane as the primary mirror. Such kind of primary mirror is characterized by poor quality, but lower weight and lower cost. The primary mirror of poor quality is the main source of phase distortions. Phase conjugation of laser radiation is used in the system to correct wave front distortions caused by aberrations of the mirror.

It should be noted that the system is not a self-directing system. The system does not use radiation incoming from a remote object for generating the PC signal. Otherwise, the system becomes very sensitive to energy of incoming radiation. The supposed system is based on technology of the telescope system with nonlinear compensation of distortions. ${ }^{9)}$ In the supposed system, the special signal to be conjugated is formed. In this case, the system operating in energy delivery mode is independent of incoming energy level.

The signal to be conjugated passes through optical system and collects information about aberrations. The signal interacts with coherent backward pump in special nonlinear medium resulting in the record of the dynamic hologram. Forward pump reads out the hologram producing phase-conjugated beam. When passing through the optical system in reverse direction, wave front distortions are corrected and a high quality laser beam is delivered to the remote object.

Finally, the very important experimental result is high efficiency of scattering of forward pump energy $-40 \%$. That means that density grating or dynamic hologram created by BEFWM process has up to $40 \%$ diffraction efficiency. Therefore, Brillouin nonlinearity can be considered as a very promising mechanism of dynamic holography used for pulsed lasers of near infrared range. This fact prompts to use Brillouin enhanced dynamic holography technique for realization of observation mode of operation in the model of the multifunctional telescope system.

\section{Conclusions}

Possibility of phase conjugation of pulsed solid-state laser radiation via Brillouin Enhanced Four-Wave Mixing has been shown experimentally. PC fidelity is estimated to be equal to 0.62 at various types of aberrations introduced into incoming signal. Diffraction efficiency of the dynamic hologram created by BEFWM process achieves $40 \%$.

Technique based on BEFWM process is considered to be applied to development of both delivery and observation modes of operation of multifunctional telescope system of effective energy delivery to various remote objects.

\section{Acknowledgements}

We would like to thank Dr. H. Yoshida for very useful discussion.

\section{References}

1) A. M. Scott and K. D. Ridley: IEEE J. Quantum. Electron. 25 (1989) 438.

2) N. F. Andreev, V. I. Bespalov, A. M. Kiselev, A. Z. Matveev, G. A. Pasmanik, and A. A. Shilov: Sov. Phys. JETP Lett. 32 (1981) 625.

3) J. R. Ackerman and P. S. Lebow: J. Opt. Soc. Am. B. 8 (1991) 1028.

4) J. R. Ackerman and P. S. Lebow: IEEE J. Quantum. Electron. 25 (1989) 479

5) K. D. Ridley and A. M. Scott: Opt. Lett. 15 (1990) 777.

6) V. Kmetik, H. Fiedorovicz, A. A. Andreev, K. J. Witte, H. Daido, H. Fujita, M. Nakatsuka, and T. Yamanaka: Appl. Opt. 37 (1998) 7085.

7) V. Kmetik, T. Kanabe, H. Fujita, M. Nakatsuka, and T. Yamanaka: Rev. Laser Eng. 26 (1998) 322.

8) O. Svelto: Principles of lasers (Plenum Press, New York, 4-th edition, 1998) p. 478.

9) M. V. Vasil'ev, V. Yu. Venediktov, A. A. Leshchev, and P. M. Semenov: Quantum Electron. 23 (1993) 267. 